\title{
Early-Onset Alzheimer's Disease and Metabolic Dysfunction, a Comparative Review
}

\author{
Julie Anne Griffith, Marie Kelly-Worden \\ Department of Biology, Ball State University, Cooper Life Science Building, Muncie, IN, USA \\ Email: mlkellyworde@bsu.edu
}

How to cite this paper: Griffith, J.A. and Kelly-Worden, M. (2018) Early-Onset Alzheimer's Disease and Metabolic Dysfunction, a Comparative Review. Advances in Alzheimer's Disease, 7, 1-11. https://doi.org/10.4236/aad.2018.71001

Received: February 28, 2018

Accepted: March 27, 2018

Published: March 30, 2018

Copyright $\odot 2018$ by authors and Scientific Research Publishing Inc. This work is licensed under the Creative Commons Attribution International License (CC BY 4.0).

http://creativecommons.org/licenses/by/4.0/

(c) (i) Open Access

\begin{abstract}
Alzheimer's disease is quickly becoming one of the most known diseases in the country due to its devastating effects and lack of treatment options. Within this lethal disease, there is a smaller group, those individuals that are diagnosed with early-onset Alzheimer's disease. It is understood that these individuals see faster effects of the disease and die considerably sooner, but it is not understood why. This review compares the early-onset (EOAD) and late-onset (LOAD) types to try and determine some of the most blaring differences between the two. The genetic basis linking EOAD and LOAD has been the apolipoprotein E gene (APOE) to indicate metabolic alteration with the $\varepsilon 4$ allele specifically. The topographical atrophy disparities between EOAD and LOAD supported the more noticeable cognitive differences between the two Alzheimer's disease categories. The faster and wider spread atrophy of EOAD patients correlates with the difficulty they experience with attention, language, visuo-spatial, and executive functions. Finally, brain metabolism differs between both $\mathrm{AD}$ subtypes as well, where EOAD indicates the wide spread damage and metabolic breakdown across more diverse regions of the brain.
\end{abstract}

\section{Keywords}

Alzheimer's Disease, Early-Onset, Late-Onset, Glucose Metabolism

\section{Introduction}

Alzheimer's disease is an age-related neurodegenerative disorder and is seen in an estimated 35 million people worldwide [1]. It is estimated that in 30 years, the United States will see 15 - 20 million total cases of some cognitive disability [2]. Although this disorder is usually recognized with old age, there are uncommon cases seen in adults under the age of 65. These cases are called early-onset Alz- 
heimer's disease (EOAD), this group represents about 10\% of all Alzheimer's cases [3].

Differences clearly exist between the two groups. In late-onset Alzheimer's disease (LOAD), adults over the age of 65 show the characteristic decline in memory function and some mood swings. This then escalates over time to the patient being unable to properly control their own body. Unfortunately, in EOAD an estimated $30 \%$ of the patients will experience aphasia, apraxia and agnosia before memory disturbances [4]. It has also been reported that EOAD patients have a more rapid clinical decline and shorter survival period after diagnosis than LOAD patients [5]. Early-onset AD patients also show greater impairment in attention, language, visuo-spatial and executive functions. It should be noted that in neuroimaging studies, these same patients demonstrate more cortical atrophy, hypoperfusion, and hypometabolism [5]. These issues mostly occur in the parietal and lateral temporal cortices of the EOAD patient, whereas LOAD patients demonstrate these issues in the medial temporal cortices [3].

When the hallmarks of Alzheimer's disease are viewed: Amyloid Beta (A $\beta)$ plaque and tau tangles, the EOAD and LOAD patients differ still. In the EOAD patients lower cerebrospinal fluid levels of $\mathrm{A} \beta$ plaque and very high total tau levels were displayed. These results were found with brain imaging techniques [3]. It should be noted that in a post-mortem study, it was found that $\mathrm{A} \beta$ burden for EOAD patients was exceedingly high like the tau [4]. Due to the previously mentioned differences in symptomology and presentation of the disease state, this paper will look to see if there is a difference in metabolic dysfunctions in EOAD patients as compared to LOAD patients.

\section{Literature Review}

\subsection{Genetic Basis in Early Onset and Late Onset Alzheimer's}

It is currently believed that less than one percent of EOAD cases are caused by an autosomal dominant mutation. The genes that account for these familial cases are PSEN1, PSEN2, and APP (6). The familial cases account for a very small proportion of the early onset patients. More commonly seen is the APOE $\varepsilon 4$ allele in sporadic AD cases. Flier stated, $50 \%-60 \%$ of individuals with $\mathrm{AD}$ are $\varepsilon 4$ carriers [6]. Those that are homozygous for the allele may develop EOAD up to 10 years earlier than individuals without the allele.

APOE in the brain is mostly produced by astrocytes and microglia. It functions as a ligand receptor-mediated endocytosis of lipoprotein particles [7]. There are three major isoforms of APOE: APOE2, APOE3, and APOE4. Kim indicated there is a strong association between the APOE gene and the presence of $\mathrm{A} \beta$ within the brain [7]. It's believed that APOE may be an $\mathrm{A} \beta$-binding protein that induces the conformational $\beta$-sheet change seen in $A \beta$ plaque, especially the APOE $\varepsilon 4$ allele. The same article also mentions the possibility of the $\varepsilon 4$ allele being linked to metabolic alterations within the brain. The $\varepsilon 4$ allele has been linked to increased levels of LDL and is a risk factor for cardiovascular disease [7]. Due 
to its link with atherosclerosis, this can cause decreased blood flow and altered metabolic properties to an inflicted individual's brain. Kim discusses a positron study carried out on young and old non-dementia patients that were carriers for the APOE $\varepsilon 4$ isoform [7]. The study found these individuals displayed similar regional patterns of hypometabolism seen in patients with $\mathrm{AD}$ of the same ages. Kim suggests that this could mean the APOE $\varepsilon 4$ is linked to the brain metabolism decrease seen in $\mathrm{AD}[7]$.

In a study carried out by Chartier-Harlin and colleagues, the APOE $\varepsilon 4$ allele presence was compared between early-onset and late-onset Alzheimer's patients and controls for the same age groups [8]. One curious concept found was that the frequency of the APOE $\varepsilon 4$ allele in EOAD and LOAD cases was not significantly different. They also found that there was an inverse correlation between the age of onset and $\varepsilon 4$ allele copy number for late-onset. This was not the case for early-onset cases. The more allele copies an individual in the LOAD category had, the earlier their onset occurred. Whereas the EOAD, it didn't seem to matter the number of $\varepsilon 4$ alleles they contained, their onset age was all relatively the same [8].

\subsection{Topographical and Cognitive Disparities}

In a study carried out by Ishii and colleagues, the local gray matter concentration between early-onset $\mathrm{AD}$ and late-onset $\mathrm{AD}$ was compared, along with controls at the same age [9]. When data was viewed, they found significant atrophy in the bilateral medial temporal lobes. Ishii notes there were greater differences between the early-onset group and their controls than the late-onset group [9]. The EOAD had less gray matter density in the bilateral inferior parietal lobules, precuneus, perisylvian cortices, and basal forebrain region. The right inferior frontal gyrus lacked gray matter density when compared to the control for the early-onset age group. Early-onset demonstrated lower densities in the bilateral precuneus, left parietal cortices, right middle temporal gyrus, and left fusiform gyrus [9].

In a study carried out by Frisoni and colleagues, they also compared the grey matter atrophy of EOAD and LOAD patients [10]. They tested a few other parameters such as neuropsychological tests and cortical mapping as well. For the neuropsychological tests, Frisoni and colleagues, showed that the early-onset patients did worse in tests that entailed visuospatial, frontal-executive, and learning concepts [10]. They also found the total gray matter atrophy in EOAD patients was greater than in LOAD, similar to the results found by Ishii. It should be noted, that atrophy for LOAD patients was higher in the left hippocampus [10]. The greatest loss for gray matter occurred in the occipital lobe for EOAD patients and in the hippocampus for LOAD patients. These results were found using volumetry, a volumetric analysis, and were later supported by the results this group found using cortical mapping techniques [10]. Figure 1 compiles Frisoni and Ishii's work and compares the grey matter atrophy of early-onset patients 
and their control counterparts. Figure 2 compiles the same data and compares late-onset versus their aged control group.

In a study by Bird, postmortem levels of choline acetyltransferase activity in the brains of EOAD, LOAD, and age-matched controls were viewed [11]. Brains were removed, one half was used for immediate pathological evaluations, and the other half was frozen. The brains riddled with Alzheimer's disease demonstrated extreme brain atrophy. Slides were made from cortex and stained with $\mathrm{H}$ and E, Holems, and periodic acid-Schiff stains. This was done to view density of plaques and tangles and to view the general distribution throughout the brain to assign disease severity. A biochemical analysis was then done on sections of the

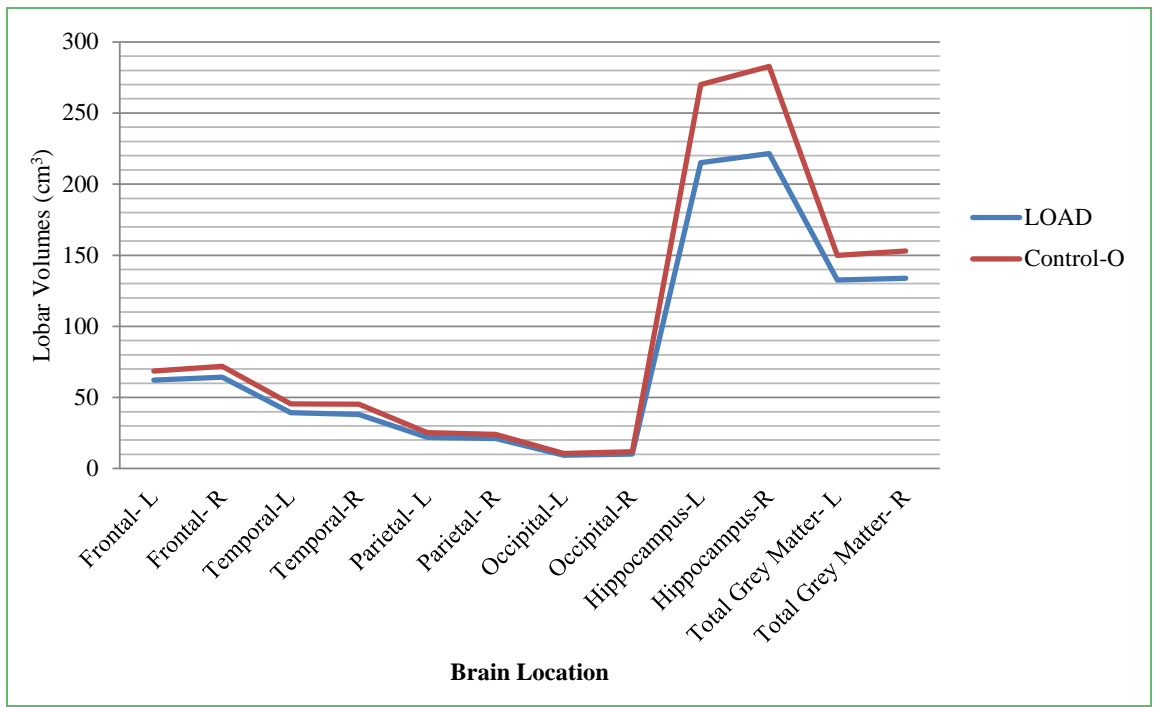

Figure 1. The information shown above demonstrates the amount of atrophy seen in late onset Alzheimer's patients compared to their same aged counterparts. Data was obtained from Frisoni and Ishii studies on grey matter atrophy [9] [10].

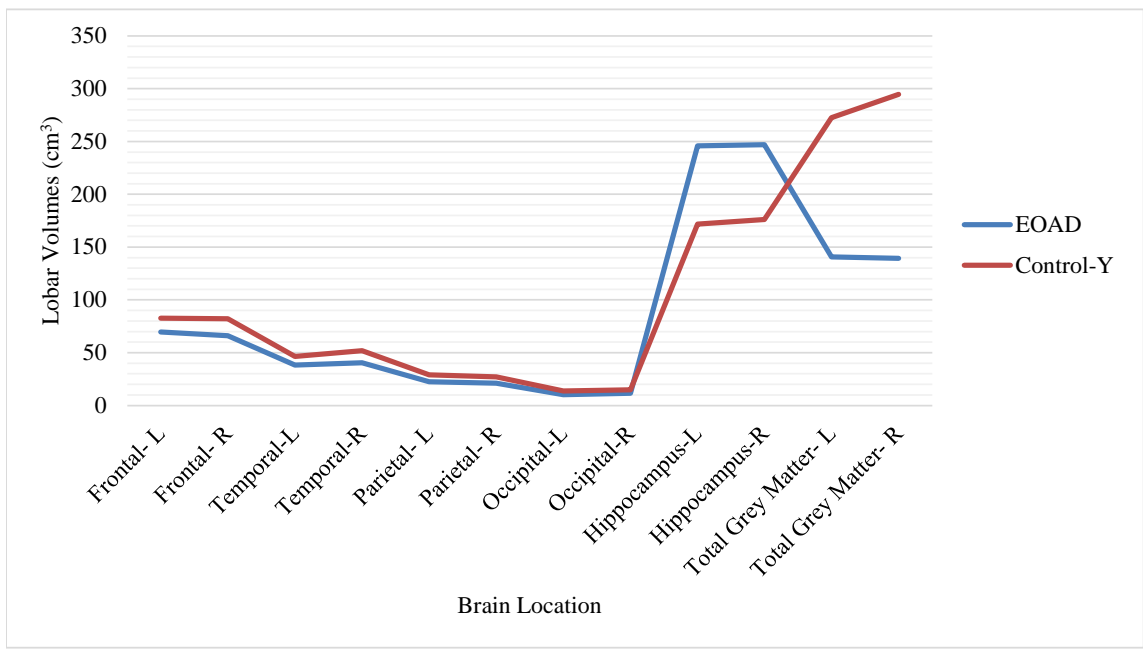

Figure 2. The information compiled compares early-onset Alzheimer's patient's brain volume to their same aged controls. Data for this diagram was used from Frisoni and Ishii studies [9] [10]. 
frozen brain from the frontal cortex, superior temporal gyrus, Ammon's horn, putamen, and lateral cerebellar hemisphere [11]. Bird found that patients with early-onset $\mathrm{AD}$ showed significantly lower choline acetyl-transferase activity than age-matched controls in the frontal cortex, temporal cortex, hippocampus, and cerebellum. Whereas the late-onset AD patients demonstrated a significant decrease in choline acetyl-transferase levels in the hippocampus [11]. These neurochemical findings support results seen in Frisoni's study [10].

In a rat model study of $\mathrm{AD}$, the metabolism in the rat brain was followed to determine the neurochemical outcome through the course of the disease. This study found that impaired neuronal mitochondria metabolism in the hippocampus, frontal cortex, frontal cingulate cortex, and retrosplenial cingulate cortex where a deceased amount of glutamate, GABA, and aspartate production occurred [12]. This reduced turnover suggests reduced TCA cycle flux in glutamatergic and GABAergic neurons in these rats. This supports the decreased glutamate levels in the cingulate cortex as seen in another study on $\mathrm{AD}$ patients [13]. They also showed decreased GABA and aspartate levels in postmortem brains which further support this, as discussed previously [12].

\subsection{Glucose Metabolism and Metabolic Differences}

Studies that previously compared cerebrospinal fluid (CSF) levels of $\beta$-amyloid have been inconsistent in the past, they may either find no significant change, a slight decrease, or increase [14]. In Andreasen and colleagues study they analyzed the cerebrospinal fluid (CSF) levels of $\mathrm{A} \beta_{(1-42)}$ and found a positive correlation between age and the CSF- $\beta$-amyloid level [14]. When compared within the Alzheimer's patients, the CSF-A $\beta$ level was significantly lower in EOAD than LOAD individuals. This study followed patients and tested their levels again at ten and twenty months. In all Alzheimer's patients, there was no significant difference between CSF-A $\beta$ levels at the baseline and ten month follow up. This study also compared the duration of dementia and their CSF levels, in which they found no significant correlation for this [14].

It should be noted that in Chiaravalloti and colleague's study, they found some different results in the cerebrospinal fluid samples [3]. In this study, Chiaravalloti and colleagues compared the levels of $\mathrm{A} \beta_{(1-42)}$ and tau. It was discovered that $\mathrm{EOAD}$ and LOAD patients had comparable levels for $\mathrm{A} \beta_{(1-42)}$ and tau, except for $\mathrm{t}$-tau. The levels of $\mathrm{t}$-tau were a little higher in the EOAD group [3].

Cerebrospinal fluid is an important part of the brain, but blood flow is another measurable factor that needs to be considered. Within the brain, blood flow is what provides the needed oxygen and glucose used by the neurons to survive [15]. Without it high amounts of cell death or dysfunction are observed, as is seen in stroke patients. Patients with AD show a few changes in brain microcirculation like appearance of string vessels (collapsed membrane tubes), reduced capillary density, mitochondrial content decrease, loss of tight junctions, and blood brain barrier breakdown. Zlokovic states, individuals with $\mathrm{AD}$ have a de- 
crease in GLUT1 expression at the Blood Brain Barrier (BBB) which leads to a shortage of necessary metabolic substances [15].

In another study that followed vascular dysregulation, Iturria-Medina and colleagues, tested for over 230 biomarkers seen in LOAD patients and sorted them according to their abnormality [16]. They discovered Cortisol, Apolipoprotein A (helps with lipid metabolism), and interferon- $\gamma$-induced protein 10 (IP-10) demonstrated some of the highest abnormalities [16]. IP-10 alterations reflect peripheral inflammation, characteristic of neurodegenerative disorders, but it is also a modulator of angiogenesis. This information provides insight into the amount of vascular dysfunction seen in very early stages of Alzheimer's.

Some research has shown that cognitive dysfunction can be linked to high-sugar/fat diet and a sedentary lifestyle [17]. In the study by Stranahan and colleagues, rats were fed a diet high in fat and simple sugars, water was replaced by high-fructose corn syrup [17]. This diet caused an increase in fasting blood glucose, serum cholesterol, and triglyceride levels. This diet impaired rat's hippocampus-dependent learning abilities, synaptic plasticity, and the dendritic spine density [17]. These rats were tested eight months into the diet and were found to perform very poorly in the water maze compared to the control rats. They were more likely to take the longer route to the platform and took them in general longer to get to the hidden platform. Hippocampi were taken from sacrificed rats and the levels of BDNF, a neurotrophic factor regulated by dietary energy, were measured. Rats on the high sugar/fat diet had reduced BDNF in whole hippocampi homogenates, which it suggests synaptic loss [17]. This leads experts in the area to conclude that there may be a link between obesity and AD as well as a link between cognitive dysfunction and abnormal glucose homeostasis.

A study done on patients with Type 1 or 2 diabetes mellitus displayed significant memory impairment and attention deficits on cognitive testing compared to their controls [18]. Hyperglycemia leads to cognitive dysfunction in a number of possible ways. The excess glucose can activate the polyol pathway, which in hyperglycemia will increase consumption of NADPH [19]. The increased use of NADPH will deplete glutathione levels and lower the threshold for oxidative injury in neurons. Klein and Waxman found increased formation of glycosylation contributed to vascular damage [19]. Any of these can alter brain function via dysfunctional blood flow, vascular permeability, or end-organ injury.

Baker and colleagues carried out a similar study on 23 adults with no previous diagnosis of prediabetes or diabetes [20]. The adults had a glucose tolerance test done and fell into one of the two categories. None met the criteria for mild cognitive impairment. Patients were given an injection of Fludeoxyglucose F-18 (FDG), and the emission was scanned for 40 minutes after the injection. For 35 minutes of the scan, patients were provided a memory encoding task, a repeating list of 20 words [20]. Adults that had prediabetes or diabetes demonstrated reduced cerebral glucose metabolic rate in brain regions commonly afflicted by 
early Alzheimer's disease. The Positron Emission Tomography (PET) scan in these adults showed a decrease in glucose metabolic rate in the posterior cingulate cortex, precuneus region, parietal cortices, temporal gyri, and prefrontal cortices [20].

A positron emission tomography (PET) study on EOAD and LOAD patients viewed regional cerebral metabolic patterns [21]. To measure the cerebral glucose metabolic rate, CMRGlu, AD patients were given an IV injection of $2\left[{ }^{18} \mathrm{~F}\right]$ fluoro-2-deoxy-D-glucose. On a PC-384 scanner, slices of the whole brain were taken and used to measure CMRGlu based on tissue activity [21]. It was found that the left temporal-parietal factor, which is involved in language cognition, processing, and comprehension, had the highest correlation to dementia severity in both EOAD and LOAD. When early-onset and late-onset were compared, this study found the right temporal-parietal factor to have significantly lower cerebral metabolism [21].

In a study carried out by Kim and colleagues, a PET scan on participants compared the hypometabolic brain patterns in patients [22]. They found the LOAD group to have more hypometabolic issues in the right inferior temporal gyrus. Whereas the EOAD had hypometabolism in the frontal lobe, basal ganglia, thalamus, and the temporoparietal areas. This same pattern of extensive overall brain complications was seen in grey matter atrophy and neurochemical pattern changes as well [22]. This shows there is a greater reduction in cerebral glucose metabolism in early-onset $\mathrm{AD}$ patients compared to their late-onset counterparts. This study also compared glucose metabolism to see if it had an effect on dementia severity. They found hypometabolism of EOAD was mild at clinical dementia rating (CDR) 0.5 , but became more widespread at higher CDR levels. When this was compared to LOAD patients, the metabolic reduction was mild until CDR 2 [22]. Figure 3 demonstrates the disparities observed in glucose hypometabolism as observed by Kim et al., 2005 and Rabinovich et al., 2010.

These findings demonstrate differences associated with glucose metabolism. Taken together with the previous data, the results here point to early-onset Alzheimer's disease as being an overall more wide-spread and faster paced disease than the late-onset version. This was demonstrated in the results obtained from previous studies that viewed the grey matter atrophy, the neurochemical changes, metabolic dysfunction, and glucose metabolism changes due to the metabolic dysfunction.

\section{Discussion}

Alzheimer's is believed to have many causes, a genetic basis, Amyloid Plaque and tau Tangles formation, or hypometabolism in the brain. All are thought to play a part in the more uncommon Early-Onset Alzheimer's Disease. This review compared these parameters, and a few others to outline the differences between $\mathrm{LOAD}$ and $\mathrm{EOAD}$, or see if there really is much of a difference.

The APOE $\varepsilon 4$ allele is indicative that an individual is more likely to develop 


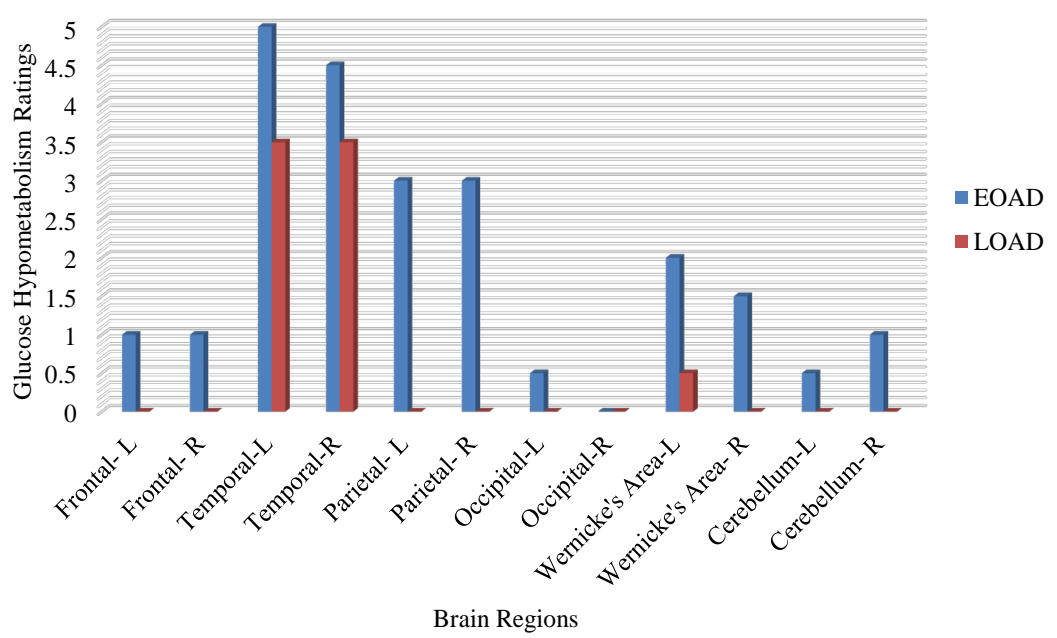

Figure 3. In the above diagram, data from Kim and colleague's study and Rabinovici and colleague's study was used [5] [22]. Both used different labeling techniques for PET scans to measure disparities in glucose hypometabolism. Scaling was based off of these images coloring patterns, with dark coloration being a 5 and the region being grey rates as a 0 .

Alzheimer's disease. This specific allele has been linked to conformational changes of the $\mathrm{A} \beta$ proteins, and causes them to clump together and diminish its role in neural growth and repair. The $\varepsilon 4$ allele has been linked to metabolic alterations in the brain via increased low density lipoproteins (LDLs). This increase has been previously known to cause atherosclerosis, which affects blood flow, in this case to the brain. This same allele is not super indicative of when an individual will develop the hallmark signs of $\mathrm{AD}$, but it is more common for individuals with this allele to have $\mathrm{AD}$.

Differences in the atrophy and chemicals that occur in EOAD and LOAD brains supports the view that EOAD is a faster paced form of Alzheimer's disease. Since EOAD patients have a difficult time with attention, language (including aphasia and apraxia), visuo-spatial, and executive functions, the locations of their greatest brain atrophy makes sense. It was shown that the greatest amount of atrophy for EOAD was in the bilateral inferior parietal lobes, occipital lobe, and basal forebrain. These same areas also demonstrated low levels of acetyl-transferase and GABA.

Some of the most trademark signs for the Late-Onset patients are their disappearing memory and mood swings. Their greatest location of atrophy and neurochemical dysfunctions was found in the hippocampus. This makes sense due to the hippocampus's main role in emotion regulation, recalling long-term memories, and spatial navigation.

One of the newest theories is that $\mathrm{AD}$ may be linked to a decrease in sleep. Studies done in rodents support this idea since wakefulness resulted in the accumulation of $\mathrm{A} \beta$. Likewise, NREM sleep supported glymphatic clearance of $\mathrm{A} \beta$ [23]. Just as likely, is the current theory involving hypometabolic dysfunction 
which has been related to high fat and sedentary lifestyles. Glucose is needed by the brain as the main source of energy. Without glucose the cells begin to die. When certain areas of the brain begin to experience microcirculation dysfunction, there is a lack in the needed glucose and metabolites. The Late-Onset individuals demonstrated hypometabolic dysfunction in the right inferior temporal gyrus, which is close to the hippocampus. The Early-Onset individuals demonstrated a more wide spread problem with hypometabolism. They found issues in the right temporal-parietal region, frontal lobe, thalamus, and basal ganglia. All of which are regions that have previously shown atrophy and neurochemical flaws in $\mathrm{AD}$.

\section{Conclusion}

This review has demonstrated that Early-Onset Alzheimer's, although a more uncommon form, is a more wide spread disease. When compared to the Late-Onset variant, EOAD demonstrates a broader array of complications that is more wide spread throughout the brain. This includes more damages due to atrophy across the entire brain, not just specific regions. The metabolic dysfunction and metabolism differences also demonstrated more wide spread metabolic dysfunction. Thus, this causes these patients to die much sooner after diagnosis than their Late-Onset counterparts. All of these patients demonstrate similar problems, just in different locations throughout the brain. More research needs to be done in the area of metabolic dysfunction for EOAD, and for all Alzheimer patients in general.

\section{References}

[1] Lu, Y., Ren, J., Cui, S., Chen, J., Huang, Y., Tang, C., Shan, B., Nie, B. and Xinsheng, L. (2016) Cerebral Glucose Metabolism Assessment in Rat Models of Alzheimer's Disease: An F-FDG-PET Study. American Journal of Alzheimer's Disease and Other Dementias, 31, 333-340. https://doi.org/10.1177/1533317515617725

[2] Mosconi, L. (2005) Brain Glucose Metabolism in the Early and Specific Diagnosis of Alzheimer's Disease. European Journal of Nuclear Medicine and Molecular Imaging, 32, 486-510. https://doi.org/10.1007/s00259-005-1762-7

[3] Chiaravalloti, A., Koch, G., Toniolo, S., Belli, L., Lorenzo, F.D., Gaudenzi, S., Schillaci, O., Bozzali, M., Sancesario, G. and Martorana, A. (2016) Comparison between Early-Onset and Late-Onset Alzheimer's Disease Patients with Amnestic Presentation: CSF and ${ }^{18}$ F-FDG PET Study. Dementia and Geriatric Cognitive Disorders EXtra, 6, 108-119. https://doi.org/10.1159/000441776

[4] Ossenkoppele, R., Zwan, M.D., Tolboom, N., Assema, D.M.E., Adriaanse, S.F., Kloet, R.W., Boellaard, R., Windhorst, A.D., Barkhof, F., Lammertsma, A.A., Scheltens, P., van der Flier, W.M. and van Berckel, B.N.M. (2012) Amyloid Burden and Metabolic Function in Early-Onset Alzheimer's Disease: Parietal Lobe Involvement. Brain, a Journal of Neurology, 135, 2115-2125. https://doi.org/10.1093/brain/aws113

[5] Rabinovici, G.D., Furst, A.J., Alkalay, A., Racine, C.A., O’Neil, J.P., Janabi, M., Baker, S.L., Agarwal, N., Bonasera, S.J., Mormino, E.C., Weiner, M.W., Gorno-Tempini, M.L, Rosen, H.J., Miller, B.L. and Jagust W.J. (2010) Increased Metabolic Vulnera- 
bility in Early-Onset Alzheimer's Disease Is Not Related to Amyloid Burden. Brain, a Journal of Neurology, 133, 512-528. https://doi.org/10.1093/brain/awp326

[6] Flier, W.M., Pijnenburg, Y.A.L., Fox, N.C. and Scheltens, P. (2011) Early-Onset Versus Late-Onset Alzheimer's Disease: The Case of the Missing APOE $\varepsilon 4$ Allele. Lancet Neurol, 10, 280-288. https://doi.org/10.1016/S1474-4422(10)70306-9

[7] Kim, J., Basak, J.M. and Holtzman, D.M. (2009) The Role of Apolipoprotein E in Alzheimer's Disease. Neuron, 63, 287-303. https://doi.org/10.1016/j.neuron.2009.06.026

[8] Chartier-Harlin, M.C., Parfitt, M., Legrain, S., Pérez-Tur, J., Brousseau, T., Evans, A., Berr, C., Vidal, O., Roques, P., Gourlet, V., Fruchart, J.C., Delacourte, A., Rossor, M. and Amouyel, P. (1994) Apolipoprotein E, $\varepsilon 4$ Allele as a Major Risk Factor for Sporadic Early and Late-Onset Forms of Alzheimer's Disease: Analysis of the 19q13.2 Chromosomal Region. Human Molecular Genetics, 3, 569-574.

https://doi.org/10.1093/hmg/3.4.569

[9] Ishii, K., Kawachi, T., Sasaki, H., Kono, A.K., Fukuda, T., Kojima, Y. and Mori, E. (2005) Voxel-Based Morphometric Comparison between Early- and Late-Onset Mild Alzheimer's Diseases and Assessment of Diagnostic Performance of Z Score Images. American Journal of Neuroradiology, 26, 333-340.

[10] Frisoni, G.B., Pievani, M., Testa, C., Sabattoli, F., Bresciani, L., Bonetti, M., Beltramello, A., Hayashi, K.M., Toga, A.W. and Thompson, P.M. (2007) The Topography of Grey Matter Involvement in Early and Late Onset Alzheimer's Disease. Brain, 130, 720-730. https://doi.org/10.1093/brain/awl377

[11] Bird, T.D., Stranahan, S., Sumi, S.M. and Raskind, M. (1983) Alzheimer's Disease: Choline-Acetyltransferase Activity in Brain Tissue from Clinical and Pathological Subgroups. Annals of Neurology, 14, 284-293. https://doi.org/10.1002/ana.410140306

[12] Nilsen, L.H., Witter, M.P. and Sonnewald, U. (2014) Neuronal and Astrocytic Metabolism in a Transgenic Rat Model of Alzheimer's Disease. Journal of Cerebral Blood Flow \& Metabolism, 34, 906-914. https://doi.org/10.1038/jcbfm.2014.37

[13] Fayed, N., Modrego, P.J., Rojas-Salinas, G. and Aguilar, K. (2011) Brain Glutamate Levels Are Decreased in Alzeimer's Disease: A Magnetic Resonance Spectroscopy Study. American Journal of Alzheimer's Disease and Other Dementias, 26, 450-456. https://doi.org/10.1177/1533317511421780

[14] Andreasen, N., Hesse, C., Davidsson, P., Minthon, L., Wallin, A., Winblad, B., Vanderstichele, H., Vanmechelen, E. and Blennow, K. (1999) Cerebrospinal Fluid $\beta$-amyloid $(1-42)$ in Alzheimer Disease. Archives of Neurology, 56, 673-680. https://doi.org/10.1001/archneur.56.6.673

[15] Zlokovic, B.V. (2011) Neurovascular Pathways to Neurodegeneration in Alzheimer's Disease and Other Disorders. Nature, 12, 723-738.

https://doi.org/10.1038/nrn3114

[16] Iturria-Medina, Y., Sotero, R.C., Toussaint, P.J., Mateos-Pérez, J.M., Evans, A.C. and Alzheimer's Disease Neuroimaging Initiative (2016) Early Role of Vascular Dysregulation on Late-Onset Alzheimer's Disease Based on Multifactorial Data-Driven Analysis. Nature Communications, 7, Article ID: No. 11934. https://doi.org/10.1038/ncomms11934

[17] Stranahan, A.M., Normal, E.D., Lee, K., Cutler, R.G., Telljohann, R., Egan, J.M. and Mattson, M.P. (2008) Diet-Induced Insulin Resistance Impairs Hippocampal Synaptic Plasticity and Cognition in Middle-Aged Rats. Hippocampus, 18, 1085-1088. https://doi.org/10.1002/hipo.20470 
[18] Cai, H., Cong, W., Ji, S., Rothman, S., Maudsley, S. and Bronwen, M. (2012) Metabolic Dysfunction in Alzheimer's Disease and Related Neurodegenerative Disorders. Current Alzheimer Research, 9, 5-17. https://doi.org/10.2174/156720512799015064

[19] Klein, J.P. and Waxman, S.G. (2003) The Brain in Diabetes: Molecular Changes in Neurons and Their Implications for End-Organ Damage. The Lancet, Neurology, 2, 548-554. https://doi.org/10.1016/S1474-4422(03)00503-9

[20] Baker, L.D., Cross, D.J., Minoshima, S., Belongia, D., Watson, G.S. and Craft, S. (2011) Insulin Resistance and Alzheimer-Like Reductions in Regional Cerebral Glucose Metabolism for Cognitively Normal Adults with Prediabetes or Early Type 2 Diabetes. Archives of Neurology, 68, 51-57. https://doi.org/10.1001/archneurol.2010.225

[21] Ichimiya, A., Herholz, K., Mielke, R., Kessler, J., Slansky, I. and Heiss, W.D. (1994) Difference of Regional Cerebral Metabolic Pattern between Presenile and Senile Dementia of the Alzheimer Type: A Factor Analytic Study. Journal of the Neurological Sciences, 123, 11-17. https://doi.org/10.1016/0022-510X(94)90197-X

[22] Kim, E.J., Cho, S.S., Jeong, Y., Park, K.C., Kang, S.J., Kim, S.E., Lee, K.H. and Na, D.L. (2005) Glucose Metabolism in Early Onset versus Late Onset Alzheimer's Disease: An SPM Analysis of 120 Patients. Brain, 120, 1790-1801. https://doi.org/10.1093/brain/awh539

[23] Winer, J.R. and Mander, B.A. (2018) Waking up to the Importance of Sleep in the Pathogenesis of Alzheimer's Disease. JAMA Neurology. https://doi.org/10.1001/jamaneurol.2018.0005 